\title{
First-principles study of high-conductance DNA sequencing with carbon nanotube electrodes
}

\author{
X. Chen, ${ }^{1}$ I. Rungger, ${ }^{1}$ C. D. Pemmaraju, ${ }^{1}$ U. Schwingenschlögl, ${ }^{2}$ and S. Sanvito ${ }^{1}$ \\ ${ }^{1}$ School of Physics and CRANN, Trinity College, Dublin 2, Ireland \\ ${ }^{2}$ PSE Division, KAUST, Thuwal 23955-6900, Kingdom of Saudi Arabia
}

(Received 7 September 2011; revised manuscript received 24 January 2012; published 26 March 2012)

\begin{abstract}
Rapid and cost-effective DNA sequencing at the single nucleotide level might be achieved by measuring a transverse electronic current as single-stranded DNA is pulled through a nanometer-sized pore. In order to enhance the electronic coupling between the nucleotides and the electrodes and hence the current signals, we employ a pair of single-walled close-ended $(6,6)$ carbon nanotubes (CNTs) as electrodes. We then investigate the electron transport properties of nucleotides sandwiched between such electrodes by using first-principles quantum transport theory. In particular, we consider the extreme case where the separation between the electrodes is the smallest possible that still allows the DNA translocation. The benzene-like ring at the end cap of the CNT can strongly couple with the nucleobases and therefore it can both reduce conformational fluctuations and significantly improve the conductance. As such, when the electrodes are closely spaced, the nucleobases can pass through only with their base plane parallel to the plane of CNT end caps. The optimal molecular configurations, at which the nucleotides strongly couple to the CNTs, and which yield the largest transmission, are first identified. These correspond approximately to the lowest energy configurations. Then the electronic structures and the electron transport of these optimal configurations are analyzed. The typical tunneling currents are of the order of $50 \mathrm{nA}$ for voltages up to $1 \mathrm{~V}$. At higher bias, where resonant transport through the molecular states is possible, the current is of the order of several $\mu \mathrm{A}$. Below $1 \mathrm{~V}$, the currents associated to the different nucleotides are consistently distinguishable, with adenine having the largest current, guanine the second largest, cytosine the third and, finally, thymine the smallest. We further calculate the transmission coefficient profiles as the nucleotides are dragged along the DNA translocation path and investigate the effects of configurational variations. Based on these results, we propose a DNA sequencing protocol combining three possible data analysis strategies.
\end{abstract}

DOI: 10.1103/PhysRevB.85.115436

PACS number(s): 87.14.G-, 87.80.St, 73.63.-b, 73.40.Gk

\section{INTRODUCTION}

In 1996, Kasianowicz et al. demonstrated the possibility of translocating by electrophoresis single-stranded DNA (ssDNA) and RNA molecules through nanometer-sized biological channels. ${ }^{1}$ This discovery initiated a surge of proposals for novel DNA sequencing protocols. ${ }^{2,3}$ Among the many, Zwolak et al. proposed to sort the nucleobases from each other in a sequence by measuring the transverse tunneling current across a ssDNA as this is driven through a nanopore. ${ }^{4}$ It was suggested that single base resolution is, in principle, achievable, as the differences in the electronic structure among the nucleobases may translate into distinguishable tunneling current signals. However, as the tunneling process is extremely sensitive to the distance between the electrodes and the nucleotides, one is left wondering whether the fluctuations in the transverse tunnel current, due to the fluctuations in the local geometry, may be too large to allow the identification of the various nucleotides. Furthermore, additional fluctuations may arise from the electrostatic gate action introduced by the solvent molecules. ${ }^{5,6}$ In order to overcome these intrinsic difficulties, it was further suggested to trace the current fluctuations as the ssDNA is translocated across the pore, and to use their statistical distribution in order to unambiguously recognize the electronic signatures of the various nucleobases. ${ }^{7-9}$

Experimentally, Tsutsui et al. showed that it is possible to identify single nucleotides in solution by two-probe tunneling current measurements and a thorough statistical analysis of the time-resolved current. ${ }^{10}$ In this case, the electrodes could be mechanically moved to optimize the current through a given nucleotide. As such, although the experiment proves the concept of using electrical currents for the sequencing, it does not demonstrate a working device.

Prototype sequencing devices with electrodes integrated in solid-state nanopores and nanofluidic channels have been also proposed. ${ }^{11-15}$ These have been realized experimentally by high-resolution milling-based methods for a number of metal electrodes, but it has been speculated that similar techniques could be used for CNTs with possibly higher resolution. ${ }^{13}$ On the theoretical front, the transverse tunneling conductance across nucleobases placed between two gold electrodes has been actively investigated and debated., ${ }^{4,16-21}$ Interestingly, recently some special attention has been dedicated to exploring graphene nanopore, graphene nanoribbon and carbon nanotubes (CNT) as potential electrodes materials. ${ }^{22-24}$

Despite these many works, a key question still remains largely unanswered, namely, how can one enhance the nucleotide-electrode interaction to a point where the transmigration is still possible, but the geometrical fluctuations are sufficiently suppressed to allow unambiguous single nucleotide recognition. Possible strategies for achieving this goal are based on functionalizing the electrodes with various chemical agents (including nucleobases themselves for example), which interact differently with the different nucelobases. ${ }^{20,24-26}$ Yet, the functionalization may be extremely challenging, in particular, if this needs to be selective at the length scale of the typical nanopore (a few nanometers). Here, we use a different strategy that does not involve any chemical functionalization and considers single-walled close-ended $(6,6) \mathrm{CNT}$ electrodes placed at an ultrashort distance [see Fig. 1(a)]. An important feature of these electrodes is that the benzene-like 


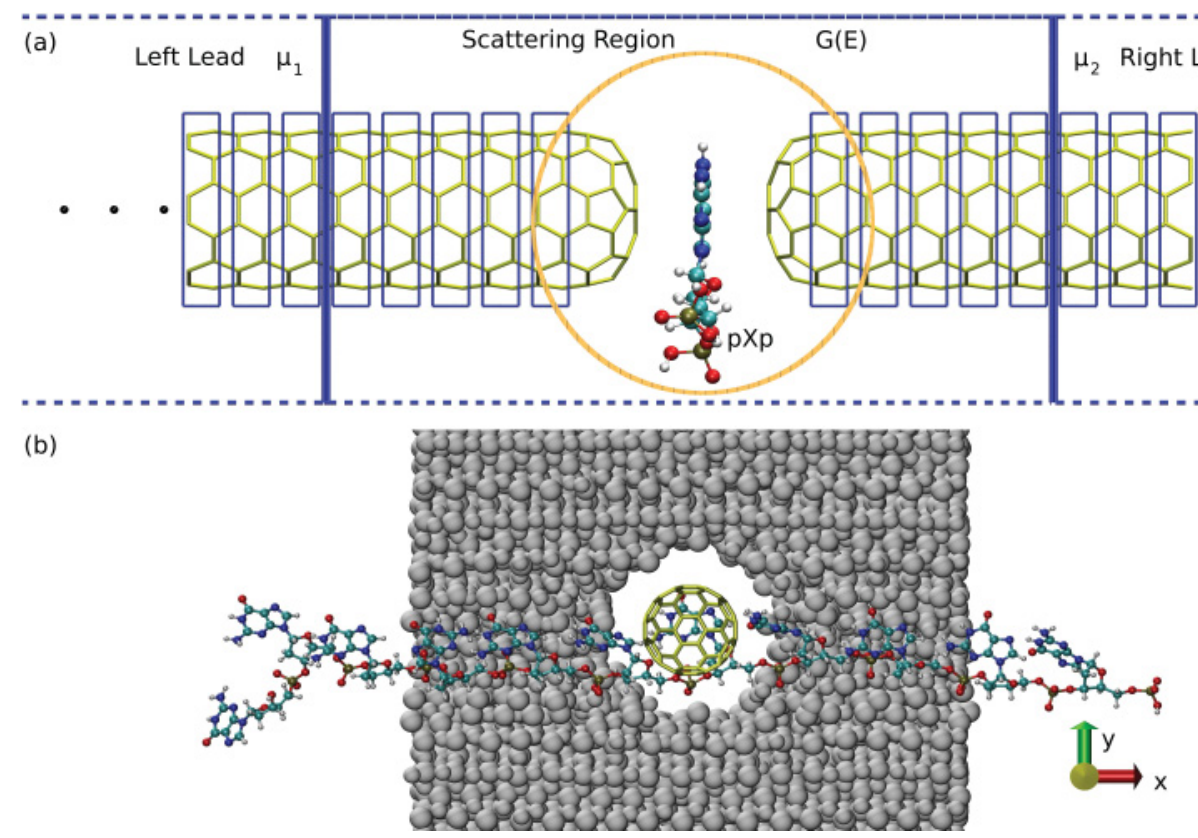

FIG. 1. (Color online) The device setup considered in this work. In (a), we present the CNT/p $X \mathrm{p} / \mathrm{CNT}(X=\mathrm{A}, \mathrm{C}, \mathrm{G}$, and T) junction used to calculate the electron transport. The left- and right-hand side $(6,6) \mathrm{CNT}$ electrodes are semi-infinite and periodic along the transport direction ( $z$ axis). In the scattering region, the $\mathrm{p} X \mathrm{p}$ molecules are sandwiched between the CNTs. A few layers of carbon atoms of both the CNTs are included in the scattering region allowing the convergence of charge density at the boundaries with the semi-infinite electrodes. In (b), a typical MD snapshot of the entire device, where the CNT electrodes are embedded into a $\mathrm{Si}_{3} \mathrm{~N}_{4}$ based nanopore [sketched by the circle in (a)], and an 11-base oligo-deoxyriboguanosine translocates through the nanopore (grey, cutaway view). The single-stranded DNAs translocate through the nanopore along the $x$ axis. A guanine base can be seen passing the gap between CNTs. Water and electrolyte ions are not displayed for clarity.

six-membered ring at the closed-end cap provides the possibility of $\pi-\pi$ coupling with the nucleobases, as ssDNA is transmigrated across. Importantly, the $\pi-\pi$ channel forces the nucleotides to align flat with respect to the electrode cap so that conformal fluctuations are highly suppressed. Our work analyzes in detail the electron transport in this favorable condition and proposes a protocol for distinguishing the nucleotides.

In short, our computational strategy unfolds as follows. We first overview the general characteristics of the transmission coefficient as a function of energy for the four nucleotides sandwiched between two such electrodes. Then we search for the optimal molecular configurations of the nucleotides about the electrodes. For these configurations, the electronic coupling is maximized and so is the low-bias current. The electronic structure, the zero-bias transmission coefficients, and the $I-V$ curves for all the nucleotides are analyzed next at the resulting optimal configurations. Finally, we calculate the transmission coefficient profiles along the full DNA translocation path and propose a DNA sequencing protocol combining multiple data analysis. A method and a conclusion section complete this work.

\section{METHOD}

The device proposed here consists of a pair of semi-infinite close-ended CNT $(6,6)$ electrodes aligned along the $z$ axis, and embedded inside a $\mathrm{Si}_{3} \mathrm{~N}_{4}$ nanopore [see Fig. 1]. The separation between the CNTs' caps is initially only $6.6 \AA$ (this will be then optimized as described later). Since the carbon van der Waals radius is about $1.7 \AA$, one may expect that the small space between the electrodes will only accommodate the planar nucleobases with their base plane parallel to the plane of CNT end caps, but not the nonplanar sugar ring and phosphate groups. This is indeed confirmed by molecular dynamics (MD) simulations performed with empirical potentials for a ssDNA pulled through a $\mathrm{Si}_{3} \mathrm{~N}_{4}$ nanopore [see the snapshot in Fig. 1(b)], from which we find that, in general, the nucleobases pass the gap between the CNTs approximately as indicated Fig. 1(a). During the translocation of a DNA strand, we expect the nucleobase to spend considerable time outside the gap between the electrodes as the aperture is rather narrow. However, as they enter the electrodes' gap, a planar configuration is always taken. Since the conductance across the electrodes is enhanced by at least seven orders of magnitude over the vacuum conductance when a nucleobase bridges the electrodes (see later), all configurations in which the bases are outside or only partially inside the electrode region, will contribute little to the signal to detect.

A detailed description of the MD simulations for the DNA translocation is outside the scope of the present paper and it will be published elsewhere. Note that here the MD is used only to verify that our electrode-electrode separation is small enough to allow the nucleobase to pass through the electrodes in a flat configuration, but it is not used to create the geometries investigated. We note that not only does such a setup minimize the structural fluctuations during translocation, but it also leads to an effective $\pi-\pi$ bonding and to a strong electronic coupling between the nucleotides and both the electrodes. We therefore expect a much larger current than that for systems where the 
nucleotides are oriented perpendicular to the electrodes, i.e., for the setup considered in several other studies. ${ }^{20,23,24}$

Although we do not pretend to offer a full fabrication strategy here, we suggest that the proposed device can be constructed by first embedding a CNT within a $\mathrm{Si}_{3} \mathrm{~N}_{4}$ membrane (perhaps by first depositing the CNT on a surface and then by regrowing $\mathrm{Si}_{3} \mathrm{~N}_{4}$ over) and then by drilling the pore with a tunnel electron microscope gun. Clearly, it still remains to be proved that during such procedure the broken apertures of the CNR reconstruct in a capped shape. A second possibility is that of bringing the two electrodes to the aperture of a prefabricated nanopore by nanomanipulation. In this case, the CNT will remain on the surface and it will not be embedded in the structure. In both cases, one requires a nanopore with a minimal diameter of the order of 1-2 $\mathrm{nm}$ (typical nanopores have a "V" shape but it is the smaller diameter, where the electrodes are placed, that characterizes the device). Such confined dimension is necessary to force the nucleobases only across the electrodes.

The four nucleotides commonly found in DNA are represented by four neutral chemical species, namely, adenosine 3'-phosphate-5' -phosphate (pAp), cytidine 3'-phosphate-5'phosphate (pCp), guanosine 3'-phosphate 5' -phosphate (pGp), and thymidine 3'-phosphate-5' -phosphate (pTp). The two phosphate groups at both sides of each nucleobase are included in order to approximate the nearest chemical environment. In the initial configurations, each of the $\mathrm{p} X \mathrm{p}(X=\mathrm{A}, \mathrm{C}, \mathrm{G}$, and $\mathrm{T}$ ) molecules is sandwiched between the two CNT end caps, with the nucleobase center of mass coinciding with the origin. This is defined as the middle point of the electrode gap and the bonds connecting the sugar ring and the nucleobases aligned along the $y$ axis. We denote these geometries as the initial geometries and assume that the nucleotides translocate along the $x$ axis. The unit cell size is set so that the distance between molecules in neighboring cells is at least $20 \AA$, along both the $x$ and $y$ directions (we use periodic boundary conditions in the direction orthogonal to that of the transport, namely in the $x-y$ plane).

All the additional geometries investigated in this work are obtained by a rigid movement of the nucleobases with respect to the electrodes, starting from the initial geometries. Thus in Sec. III B the search for the configuration yielding the highest zero-bias conductance is performed by simultaneous total-energy and zero-bias transport calculations of geometries obtained from the initial geometries through translations and rotations. Finally, the investigation of the effects of configurational disorder and of the nucleobase translocation (see Secs. III E and III F) are obtained by looking at geometries obtained through rotations and translations starting from the optimal geometries.

The computation of the transmission coefficients $(T)$ and the current-voltage $(I-V)$ characteristics is performed by using the $a b$ initio electronic transport code SMEAGOL. ${ }^{27-29}$ SMEAGOL implements the nonequilibrium Green's function (NEGF) method over density functional theory (DFT), by using the pseudopotential code SIESTA $^{30}$ as its electronic structure platform. In SMEAGOL, the device under investigation is partitioned into three regions: the left- and right-hand side semi-infinite current-voltage electrodes and the scattering region [see Fig. 1(a)]. In our calculations, the scattering region comprises 156 and 144 carbon atoms, respectively, of leftand right-hand-side CNT electrodes, and one of the $\mathrm{p} X \mathrm{p}$ molecules. The portion of the electrodes included explicitly in the scattering region is large enough to ensure that the calculated charge density at the outermost layers converges to that of bulk CNTs.

Both the electronic structure and the transport properties are calculated with the local density approximation (LDA) of the DFT exchange and correlation functional. A double- $\zeta$ plus polarization basis sets is used for $\mathrm{C}(2 s 2 p), \mathrm{N}(2 s 2 p)$, $\mathrm{O}(2 s 2 p), \mathrm{P}(3 s 3 p)$, and $\mathrm{H}(1 s)$ throughout. All calculations are carried out with an equivalent real-space mesh cutoff of $300 \mathrm{Ry}$ and an electronic temperature of $300 \mathrm{~K}$. In SMEAGOL, the charge density is evaluated by separating the integral over the nonequilibrium Green's function into an equilibrium part, which is performed along a contour in the complex energy plane, and a nonequilibrium part, which is performed along the real energy axis. ${ }^{28}$ For the first, we use 16 energy points on the complex semicircle, 16 points along the line parallel to the real axis, and 16 poles. The integral over real energies necessary at finite bias is evaluated over a mesh, whose energy spacing is not larger than $1 \mathrm{meV}^{27,28}$

Note that the use of the LDA for transport calculations may be, in general, problematic because of the typical underestimation of the energy gap and the related incorrect alignment of the molecular levels with respect to the electrodes Fermi energy. Since most of these features may be attributed to the self-interaction error, ${ }^{31}$ an approximated way to introduce self-interaction corrections (SIC) was introduced in the past and used for the electron transport problem, demonstrating a better agreement with experiments. ${ }^{32,33}$ In particular a qualitative difference between LDA and LDA + SIC has been reported for situations where the LDA erroneously places a molecular level at the Fermi level, i.e., when the transport is described as resonant through a molecular orbital instead as tunneling. In contrast, when the LDA already gives us tunneling conductance, the differences are only quantitative. This is fortunately the situation encountered here as in all the configurations investigated the transport is always tunneling like. As we do not expect that the inclusion of SIC will change the relative alignment of the molecular levels of the four nucleobases with respect to the Fermi level of the CNT, we expect that the general conclusion of our work will be robust against SIC.

\section{RESULTS AND DISCUSSION}

\section{A. General characteristics of the transmission at zero bias}

We first examine the general transport properties of all the possible four $\mathrm{CNT} / \mathrm{p} X \mathrm{p} / \mathrm{CNT}(X=\mathrm{A}, \mathrm{C}, \mathrm{G}$, and $\mathrm{T})$ junctions by plotting, in Fig. 2, the zero-bias transmission coefficient as function of energy for the initial geometries, where the nucleobases' center of mass coincides with the middle point of the electrode gap. Electron tunneling between the CNT electrodes (6.6 $\AA$ apart in these initial simulations) through vacuum (we denote this configuration as CNT//CNT) is extremely small $\left(T \sim 1.3 \times 10^{-12}\right)$ around the Fermi level, $E_{\mathrm{F}}$. Note that the linear response (zero-bias) conductance, $G$, is simply $G=G_{0} T\left(E_{\mathrm{F}}\right)$, where $G_{0}=2 e^{2} / h$ is the quantum 


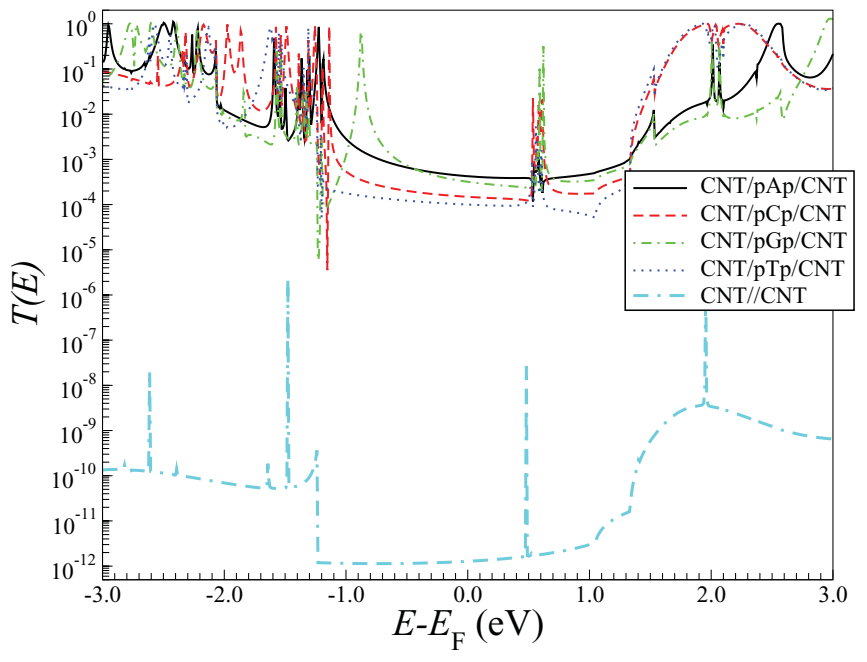

FIG. 2. (Color online) Zero-bias transmission coefficient as a function of energy for $\mathrm{CNT} / / \mathrm{CNT}$ and the four $\mathrm{CNT} / \mathrm{p} X \mathrm{p} / \mathrm{CNT}$ $(X=\mathrm{A}, \mathrm{C}, \mathrm{G}$, and $\mathrm{T})$ junctions. The curves refer to the initial configurations where the nucleobase' center of mass coincides with the middle point of the electrode gap. The CNT electrodes are $6.6 \AA$ apart.

conductance, $e$ is the electronic charge and $h$ the Planck constant, so that a tiny $T\left(E_{\mathrm{F}}\right)$ is equivalent to a small linear response conductance.

The inclusion of the $\mathrm{p} X \mathrm{p}$ molecules in the device results in an increase of the transmission coefficient by over eight orders of magnitude with typical values of $T\left(E_{\mathrm{F}}\right)$ of around $10^{-4}-10^{-3}$. We therefore expect in general a drastic change in the measured current when a nucleotide passes between the CNT electrodes, regardless of the nucleotide type. As such our device is at least capable of distinguishing whether or not a molecule is between the two electrodes. Notably, the magnitude of the transmission coefficient at $E_{\mathrm{F}}$ is found to be orders of magnitude larger than that found by using either functionalized gold $\left(\sim 10^{-6}\right.$, see Ref. 20$)$ or graphene $\left(>10^{-10}\right.$, see Ref. 23) electrodes (in both cases, the calculations have been carried out with SMEAGOL using similar computational parameters). Clearly, our much larger tunneling current originates from the short electrode-electrode separation and by the fact that the nucleobases lie flat between the electrodes.

In the various $T(E)$ profiles there are peaks, as the one clearly visible at $0.5 \mathrm{eV}$, common to all the junctions. All of these are very pronounced and extremely sharp and they are characteristic of the CNT//CNT device as well (see peaks at $-2.61,-1.64,-1.48$, and $1.95 \mathrm{eV}$, with all the energies taken with respect to the CNT $E_{\mathrm{F}}$ ). These are all due to localized surface states of the close-ended CNTs and therefore are not representative of any feature of the nucleotide electronic structure. Such CNT surface states can be readily identified by comparing the density of states (DOS) of an infinite CNT with the one of the semi-infinite close-ended CNT, shown in Fig. 3.

For all the $\mathrm{CNT} / \mathrm{p} X \mathrm{p} / \mathrm{CNT}$ junctions investigated, we can identify two distinct transport regimes (see Fig. 2): a tunneling regime for energies between -1.0 and $1.5 \mathrm{eV}$, and a resonant transport regime through the nucleotides' molecular levels for energies outside this range. Around $E_{\mathrm{F}}$, the transmission curves for all the nucleotides are very smooth and vary little

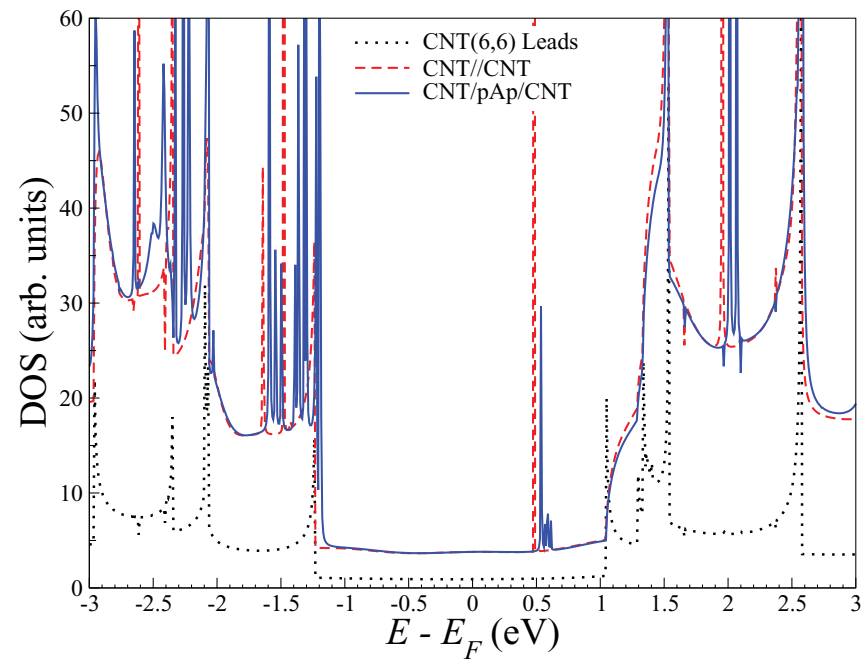

FIG. 3. (Color online) Density of states (DOS) of a $(6,6) \mathrm{CNT}$ electrode and of both the CNT//CNT and the CNT/pAp/CNT junctions as function of energy for the initial configurations. $\mathrm{CNT} / \mathrm{pAp} / \mathrm{CNT}$ is chosen as a representative of the $\mathrm{CNT} / \mathrm{p} X \mathrm{p} / \mathrm{CNT}$ junctions.

over energy. Importantly, the relative order of magnitude of the transmission coefficients, namely, $T_{\mathrm{pAp}}>T_{\mathrm{pGp}}>T_{\mathrm{pCp}}>$ $T_{\mathrm{pTp}}$, is preserved for a rather large energy range around the Fermi level. This indicates that it is possible to distinguish the nucleotides already in the low-bias tunneling regime, and that the recognition is relatively robust with respect to fluctuations in the position of $E_{\mathrm{F}}$. Furthermore, the flatness of the transmission coefficient around $E_{\mathrm{F}}$ suggests that one can infer the current for voltages up to about $1 \mathrm{~V}$ from the simple relation $I \approx T\left(E_{\mathrm{F}}\right) V$. Therefore in the next section, we will analyze the transport properties at the Fermi energy only, and search for the molecular configurations of the $\mathrm{CNT} / \mathrm{p} X \mathrm{p} / \mathrm{CNT}$ junctions that yield the largest transmission at the Fermi energy. When these configurations will be explored by the ssDNA during the translocation process, they will provide the largest contribution to the current.

\section{B. Search for the molecular configurations yielding the maximum transmission}

For the device setup investigated here, we have identified four possible degrees of freedom, which determine the relative position of the $\mathrm{p} X \mathrm{p}$ molecules with respect to the electrodes: (1) the rotation angle of $\mathrm{p} X \mathrm{p}$ around the $z$ axis [up to $60^{\circ}$ due to the $(6,6)$ CNT symmetry], (2) a translation of the $\mathrm{p} X \mathrm{p}$ molecules about the $y$ and (3) the $x$ axes, and (4) a variation of the distance between the two CNT electrodes. Here, we assume that the internal structure of the $\mathrm{p} X \mathrm{p}$ molecules does not deform and that the nucleobases remain parallel to the CNT end cap planes during the translocation.

In Sec. IIIF, we will discuss in detail the effects of these operations on the electron transport; here, we first identify the configurations that maximize the transmission at $E_{\mathrm{F}}$. To this goal, we start from the initial configurations of the $\mathrm{CNT} / \mathrm{p} X \mathrm{p} / \mathrm{CNT}$ junctions, defined in Sec. II, and perform all the possible independent rotations and translations. Interestingly and importantly, we find that the molecular 
configurations having the largest transmission coefficients are generally found to be close to those that minimize the system energy. This is due to the fact that when the $\pi-\pi$ interaction between the nucleotides and the CNTs is large, the total energy is generally minimized and the transmission maximized.

We first determine the optimal distance between the CNTs. The guiding principle for the optimization is that the transmission is expected to increase for decreasing CNT separation, but the separation needs to be large enough to allow the translocation of the nucleotides. In any hypothetical fully operational device, the CNT electrodes' position is fixed as these are embedded in the nanopore, so that we have to find a separation that is optimal for all the four nucleotides. In particular, the electrodes gap should be wide enough to allow also the nonplanar methyl group on thymine to pass through. In order to determine the optimal distance between the CNT electrodes, we then fix the molecule in the lateral position (in the $x-y$ plane) that yields the largest transmission at a CNT separation of $6.6 \AA$ and then change the separation between the electrodes, always keeping the molecule in the middle of the gap.

We examine first the junction total energy as a function of the separation between the CNT caps (see Fig. 4). The smallest possible CNT electrodes gaps can be seen to be approximately $5.8 \AA$, since there is a sharp increase of the energy for closer separations regardless of the nucleotide type. Considering that the optimal distance may be underestimated by DFT calculations in which the van der Waals interactions (which tend to be repulsive at short distances) are not included, and that we should use a common distance for all four CNT/p $X \mathrm{p} / \mathrm{CNT}$ junctions, in all subsequent calculations, we assume that the device has been set up with a distance of $6.4 \AA$ between the two CNT electrodes. For this electrodes distance, the molecular configurations of the four $\mathrm{CNT} / \mathrm{p} X \mathrm{p} / \mathrm{CNT}$ junctions yielding the maximum transmission at $E_{\mathrm{F}}$ are summarized in Table I in terms of the geometrical offset relative to their initial

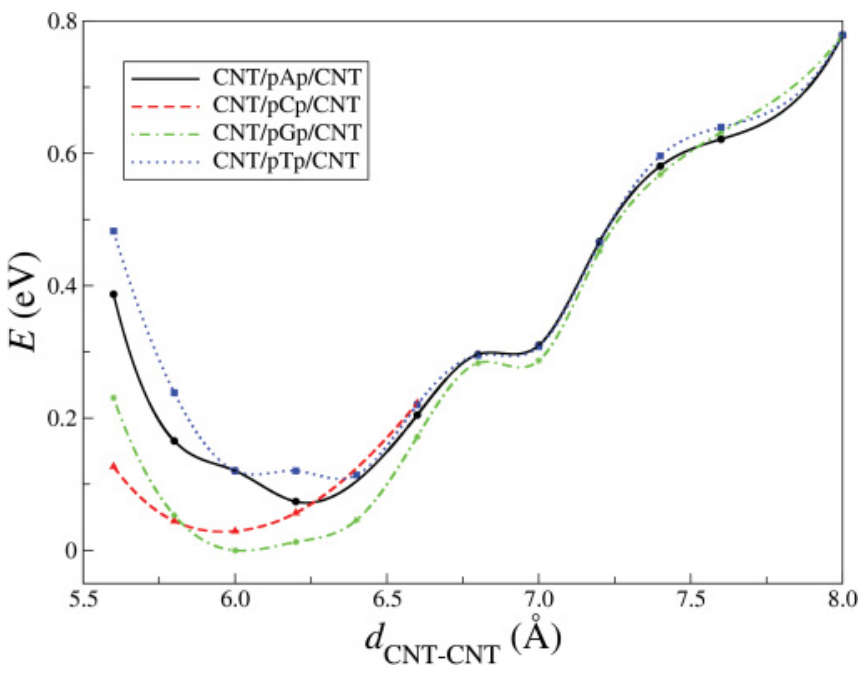

FIG. 4. (Color online) Total energy as a function of the distance between the CNT electrodes, $d_{\mathrm{CNT}-\mathrm{CNT}}$, for the CNT/p $X \mathrm{p} / \mathrm{CNT}$ junctions $(X=\mathrm{A}, \mathrm{C}, \mathrm{G}$, and $\mathrm{T})$. The energy zero is taken at the lowest energy calculated.
TABLE I. Optimal molecular configurations, which lead to maximal transmission at $E_{\mathrm{F}}$, for the four $\mathrm{CNT} / \mathrm{p} X \mathrm{p} / \mathrm{CNT}$ junctions ( $X=\mathrm{A}, \mathrm{C}, \mathrm{G}$, and $\mathrm{T}$ ), relatively to the initial configurations. $\theta$ is the right-hand rotation angle of the nucleobases about the $z$ axis. The distance between the CNTs for optimal configurations is chosen to be commonly $6.4 \AA$.

\begin{tabular}{lcccr}
\hline \hline & $\mathrm{pAp}$ & $\mathrm{pCp}$ & $\mathrm{pGp}$ & $\mathrm{pTp}$ \\
\hline$\theta$ & $14.32^{\circ}$ & $12.28^{\circ}$ & $14.28^{\circ}$ & $11.08^{\circ}$ \\
Offset $y(\AA)$ & -0.21 & -0.14 & -0.09 & 0.03 \\
Offset $x(\AA$, peak 1) & -0.05 & -2.13 & -0.58 & -1.88 \\
Offset $x(\AA$, peak 2) & $\cdots$ & 1.01 & $\cdots$ & 1.19 \\
\hline \hline
\end{tabular}

configurations. We denote these configurations as the optimal configurations.

An interesting result of our search for the optimal configurations is that $\mathrm{pCp}$ and $\mathrm{pTp}$ turn out to have two configurations where the transmission coefficient peaks (see Fig. 5 for a graphic representation of all the optimal configurations). In these, the smaller pyrimidine bases of the cytosine and thymine are located at either side of the CNT electrodes along the $x$ axis. In contrast, $\mathrm{pAp}$ and $\mathrm{pGp}$ appear to have only one major peak, and the configuration is such that the larger purine bases of adenine and guanine are located in the center of electrodes

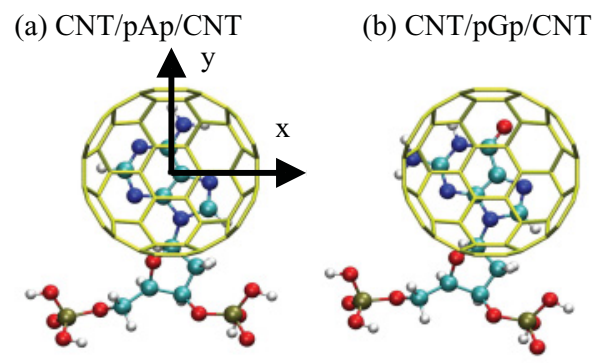

(c) $\mathrm{CNT} / \mathrm{pCp} / \mathrm{CNT}$ :peak 1

(d) $\mathrm{CNT} / \mathrm{pCp} / \mathrm{CNT}$ :peak 2
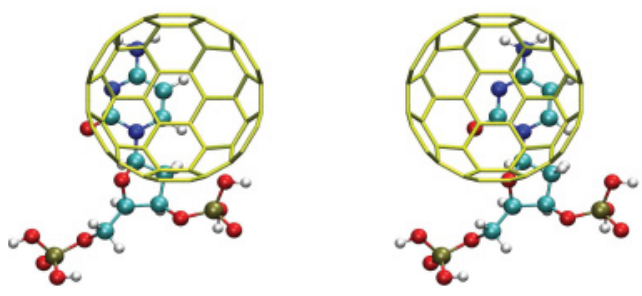

(e) $\mathrm{CNT} / \mathrm{pTp} / \mathrm{CNT}$ :peak 1

(f) $\mathrm{CNT} / \mathrm{pTp} / \mathrm{CNT}$ :peak 2
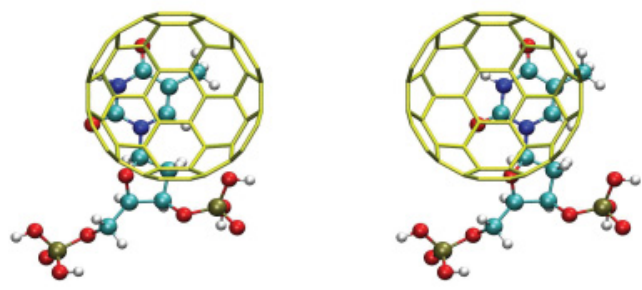

FIG. 5. (Color online) Optimal molecular configurations for the $\mathrm{CNT} / \mathrm{p} X \mathrm{p} / \mathrm{CNT}$ junctions $(X=\mathrm{A}, \mathrm{C}, \mathrm{G}$, and $\mathrm{T})$. For $\mathrm{pCp}$ and $\mathrm{pTp}$, the two equivalent configurations are both shown. 
gap. This difference between purine and pyrimidine bases results in distinct profiles of the transmission coefficient along the ssDNA translocation direction (see later in Sec. IIIE). In the calculations that will follow, we will consider always the configuration responsible for the first of the transmission peaks for the $\mathrm{CNT} / \mathrm{pCp} / \mathrm{CNT}$ and $\mathrm{CNT} / \mathrm{pTp} / \mathrm{CNT}$ junctions.

A final note must be made about the physical meaning of the optimal configurations. Indeed, these simply represent those geometrical arrangements for which the zero-bias conductance is the largest, i.e., those configurations that maximize the transmission. As such, they do not bare any particular relevance for the sequencing. However, we have found that such optimal configurations are also those that minimize the total energy, since they are the ones in which the $\pi-\pi$ interaction is the strongest. As such, assuming that the translocation of the DNA across the electrodes is guided by total energy considerations only (the kinetics of the process plays only a secondary role), we expect that the optimal configuration will be also the most probable during the translocation process.

\section{Electronic structure and zero-bias transmission at the optimal configurations}

In order to understand the details of the electron transfer across the $\mathrm{CNT} / \mathrm{p} X \mathrm{p} / \mathrm{CNT}$ ( $X=\mathrm{A}, \mathrm{C}, \mathrm{G}$ and $\mathrm{T}$ ) junctions, we now analyze the relation between the transmission and the electronic structure of the devices at their optimal configurations. In Fig. 6, we plot the zero-bias transmission coefficient and the DOS of the $\mathrm{p} X \mathrm{p}$ molecules forming the $\mathrm{CNT} / \mathrm{p} X \mathrm{p} / \mathrm{CNT}$ junctions. The vertical dashed lines indicate the energies of the eigenvalues of the isolated $\mathrm{p} X \mathrm{p}$ nucleotides, i.e., they correspond to the DOS of the isolated molecules. From the figure, it is clear that the eigenvalues of the isolated molecules align well with the peaks in the DOS of the corresponding junction [note that a global shift is applied in order to align the
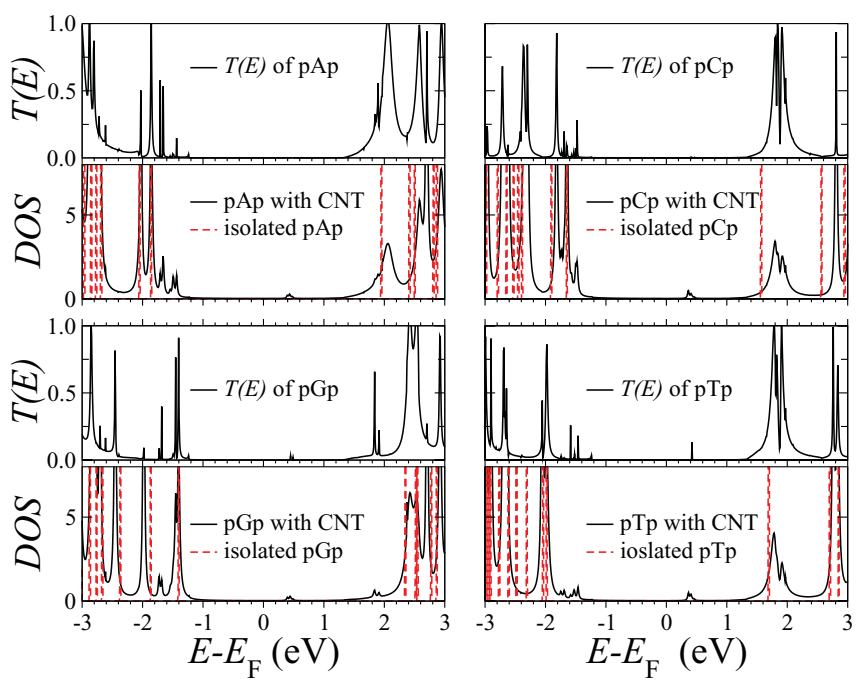

FIG. 6. (Color online) Zero-bias transmission coefficient as a function of energy and corresponding density of states (DOS) projected onto the $\mathrm{p} X \mathrm{p}$ molecules for the four $\mathrm{CNT} / \mathrm{p} X \mathrm{p} / \mathrm{CNT}$ junctions. In the panels showing the DOS, we also present, as dashed vertical lines, the DOS of the $\mathrm{p} X \mathrm{p}$ molecules in the gas phase.
TABLE II. Energy position of the HOMO and the LUMO of the $\mathrm{p} X \mathrm{p}$ molecules in their corresponding $\mathrm{CNT} / \mathrm{p} X \mathrm{p} / \mathrm{CNT}$ junctions $(X=\mathrm{A}, \mathrm{C}, \mathrm{G}$, and $\mathrm{T})$.

\begin{tabular}{lrrrr}
\hline \hline & pAp & pCp & pGp & \multicolumn{1}{c}{ pTp } \\
\hline HO $(\mathrm{eV})$ & -1.86 & -1.65 & -1.40 & -1.98 \\
LU $(\mathrm{eV})$ & 2.06 & 1.81 & 2.42 & 1.78 \\
\hline \hline
\end{tabular}

highest occupied molecular orbital (HOMO) of the molecule in the gas phase to that in the junction]. This is indicative of the fact that the molecule-electrode interaction does not involve charging, so that the molecule spectrum is not distorted in any significant way. However, the DOS of the junctions reveals a considerable level broadening, suggesting that there is a substantial overlap between the molecular orbitals of the nucleotides and the extended states of the electrodes.

This strong overlap is responsible for the high transmission and Fig. 6 reveals a one-to-one correspondence between the molecule DOS and the peaks in the transmission functions. In particular, for all the four junctions, the HOMO and the lowest unoccupied molecular orbital (LUMO), both located on $\mathrm{p} X \mathrm{p}$ molecules, are far away from the electrodes' Fermi level. As such the tunneling transmission region around $E_{\mathrm{F}}$ corresponds to the molecule HOMO-LUMO gap. A summary of the energy position of both the HOMO and the LUMO of the four junctions is presented in Table II.

At the optimal configurations (see Fig. 5) the $\mathrm{p} X \mathrm{p}$ molecules are strongly coupled to the CNT electrodes, through the optimal $\pi-\pi$ coupling of the nucleobases with the CNT end caps. In order to visualize such a $\pi-\pi$ bond, we calculate the real-space local density of states (LDOS) associated to the charge density of both the HOMO and the LUMO of the $\mathrm{CNT} / \mathrm{p} X \mathrm{p} / \mathrm{CNT}$ junctions. For the HOMO (LUMO), we integrate the charge density over an energy window $45 \mathrm{meV}$ $(50 \mathrm{meV})$ wide around the associated peak in the DOS. The results are shown in Fig. 7, where the $\pi-\pi$ bonds are clearly
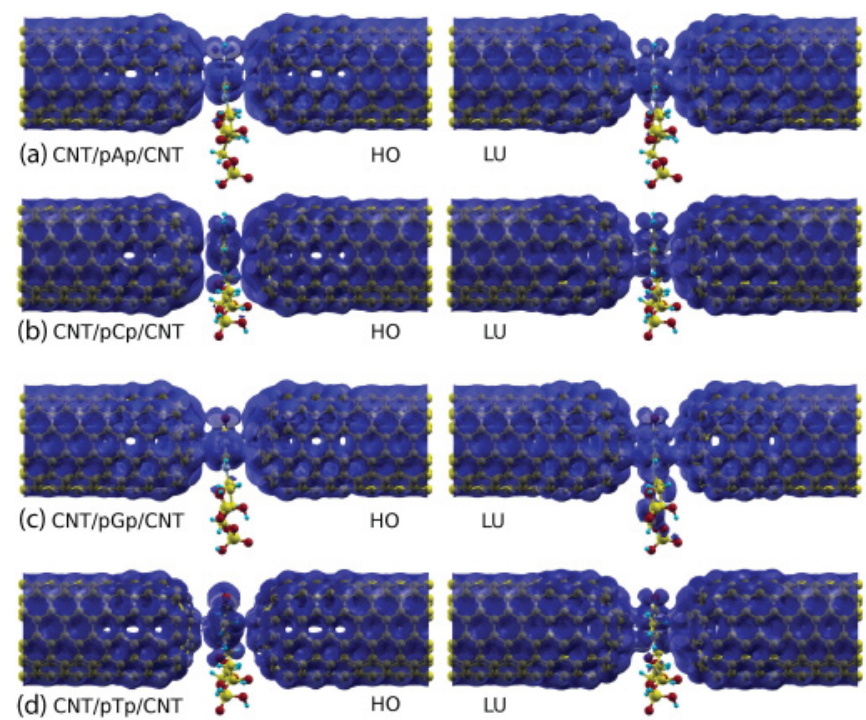

FIG. 7. (Color online) Local density of state (LDOS) isosurfaces of the HOMO and LUMO of the CNT/p $X \mathrm{p} / \mathrm{CNT}$ junctions at zero bias. The geometries corresponds to the optimal configurations. 


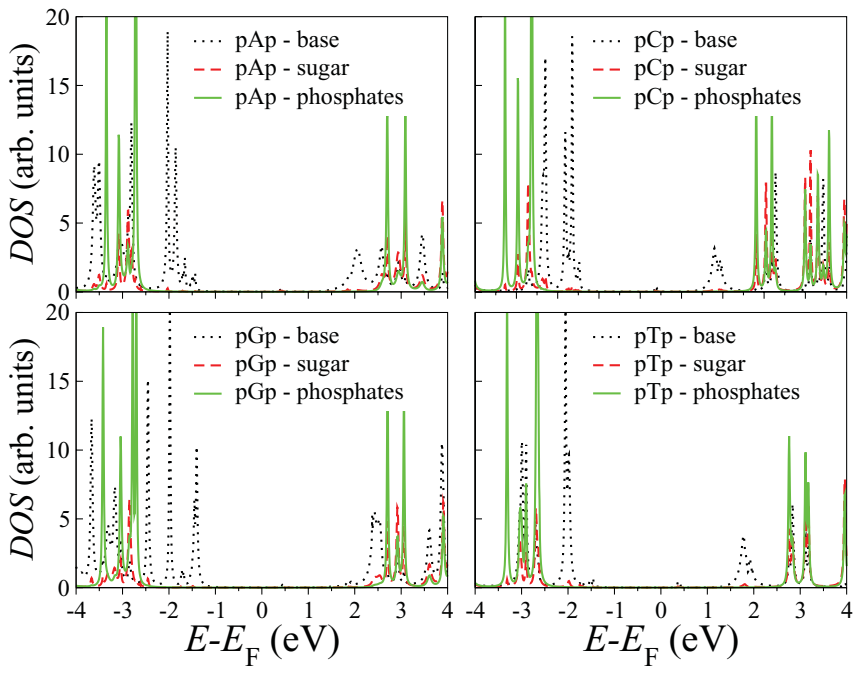

FIG. 8. (Color online) Density of states (DOS) projected onto the base, the sugar, and the phosphates of the various $\mathrm{p} X \mathrm{p}$ molecules in the $\mathrm{CNT} / \mathrm{p} X \mathrm{p} / \mathrm{CNT}$ junctions, calculated at zero bias at the optimal configurations.

visible. The face-to-face $\pi-\pi$ stacking of the six-member rings of the CNT end caps with the nucleobases not only makes the optimal configurations energetically favorable, but also enhances the electron tunneling. Since the $\pi-\pi$ interaction disappears when the nucleobases are outside the central region in between the electrodes, one may expect that the distribution of transmission coefficients (and hence the currents) should peak when the nucleotides are inside such a region and that the high transmission configurations should be rather stable.

Importantly, the $\pi-\pi$ interaction of the $\mathrm{p} X \mathrm{p}(X=\mathrm{A}, \mathrm{C}, \mathrm{G}$, and T) molecules with the CNTs involves only the nucleobases. This means that the sugar ring and two nearest phosphate groups in $\mathrm{p} X \mathrm{p}$ do not play any significant role either in the bonding or in the electron transport. A demonstration of this feature is provided in Fig. 8, where we present the DOS projected respectively onto the nuclebase, the sugar, and the phosphate groups. From the figure, it is clear that both the HOMO and the LUMO are almost entirely associated to the nuclebases, while both the sugar and the phosphate groups contribute only to molecular levels far way from the Fermi level. This is a rather important aspect of the electronic structure of the DNA/CNT system investigated here, namely, the fact that the nonsequence specific features of the DNA spectrum are away from the electrodes Fermi level and therefore are not expected to contribute significantly to the transport at least at relatively moderate voltages.

Finally, we wish to investigate further which functional part of the nucleotides contributes the most to the $\pi-\pi$ bonding by looking at the DOS projected either on the amine $\left(\mathrm{NH}_{2}\right)$ or the carbonyl $(\mathrm{C}=\mathrm{O})$ group (see Fig. 9). Note that pAp, pCp, and $\mathrm{pGp}$ have one primary amine group, $\mathrm{pCp}$ and $\mathrm{pGp}$ have one carbonyl and, finally, pTp has two carbonyls. From Fig. 9, we note that for $\mathrm{pAp}$, the primary amine group contributes the most to the HOMO, with a major peak in the DOS at around $-1.86 \mathrm{eV}$ and minor peaks at around -1.66 and $-1.44 \mathrm{eV}$. These form because of the coupling to the CNT electrodes. The presence of such minor peaks helps to explain
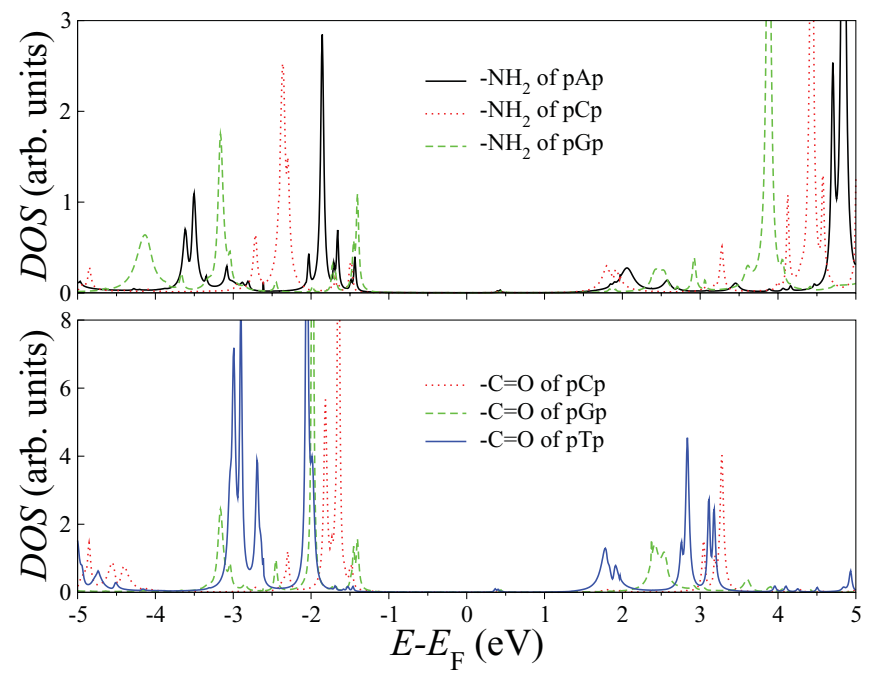

FIG. 9. (Color online) Density of states (DOS) projected onto the amine and carbonyl groups of the various $\mathrm{p} X \mathrm{p}$ molecules in the $\mathrm{CNT} / \mathrm{p} X \mathrm{p} / \mathrm{CNT}$ junctions, calculated at zero bias at the optimal configurations.

why at zero bias pAp has the largest transmission coefficient despite that its major HOMO peak lies below those of $\mathrm{pCp}$ and $\mathrm{pGp}$ (see Table II). In contrast for $\mathrm{pCp}$, the carbonyl group contributes more than the primary amine one to the $\mathrm{HOMO}$, while for $\mathrm{pGp}$, these two groups contribute similarly to their HOMO. The main peaks of the carbonyl groups appear to be similar for pGp and pTp (around $-2 \mathrm{eV}$ ) but these are located at higher energies for $\mathrm{pCp}$ (around $-1.65 \mathrm{eV}$ ). The different positioning and strength of the coupling to the electrodes of the electronic states located on these functional groups are important, since they affect the tunneling signals for the different nucleobases. Their characteristic signals may be augmented by functionalizing the electrodes with suitable chemical agents in order to amplify the electronic contrast between the different nucelobases. ${ }^{20,25,26}$

\section{D. $I-V$ characteristics at the optimal configurations}

We now investigate the transport properties of the $\mathrm{p} X \mathrm{p}$ molecules $(X=\mathrm{A}, \mathrm{C}, \mathrm{G}$, and $\mathrm{T})$ by assuming that these are ideally measured at the optimal configurations. Figure 10 shows the zero-bias transmission coefficient, $T(E)$, plotted on a logarithmic scale. As noted previously, the peaks in transmission at around $0.5 \mathrm{eV}$ are due to localized surface states belonging to the CNT close ends. These are unaffected by the $\mathrm{p} X \mathrm{p}$ configuration and they contribute little to the electron current as bias is applied (for a discussion on the resonant transport properties of surface localized state see Ref. 34). At the Fermi level, the relative order of magnitude of the transmission coefficient is now $T_{\mathrm{pAp}}>T_{\mathrm{pGp}}>T_{\mathrm{pCp}}$ $>T_{\mathrm{pTp}}$, i.e., it is the same order that we have found for the initial configurations (see Fig. 2). However, if one now looks at energies away from $E_{\mathrm{F}}$, a few changes are notable. For instance, at $-0.77 \mathrm{eV}$ (energies are always taken from $E_{\mathrm{F}}$ ), the transmission coefficient of $\mathrm{pGp}$ surpasses that of $\mathrm{pAp}$ and the order becomes $T_{\mathrm{pGp}}>T_{\mathrm{pTp}}>T_{\mathrm{pAp}}>T_{\mathrm{pCp}}$. In contrast, for positive energies, $T_{\mathrm{pTp}}$ and $T_{\mathrm{pCp}}$ first surpass $T_{\mathrm{pGp}}$ at $0.5 \mathrm{eV}$ and 


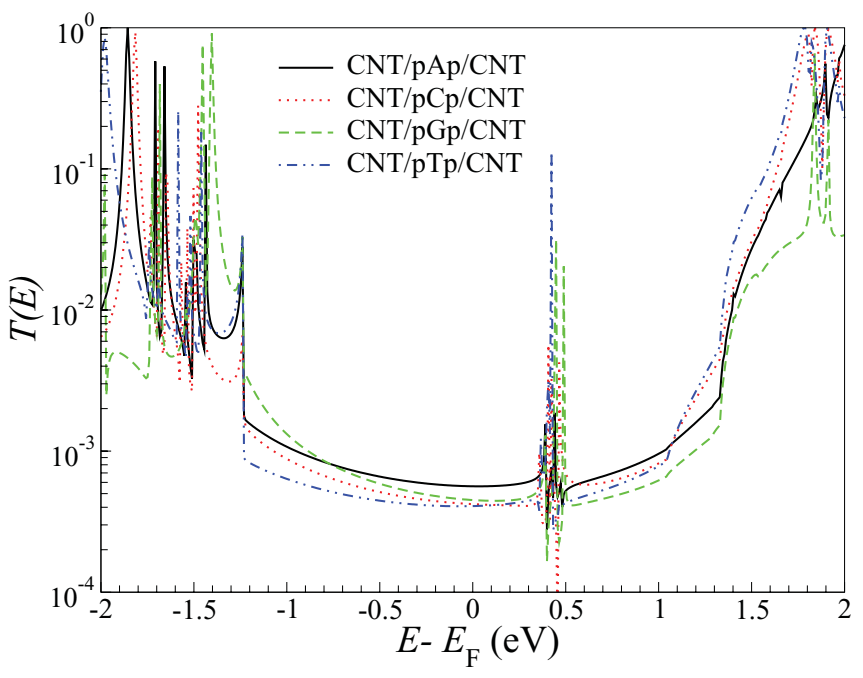

FIG. 10. (Color online) Zero-bias transmission coefficient as a function of energy for $\mathrm{CNT} / \mathrm{p} X \mathrm{p} / \mathrm{CNT}(X=\mathrm{A}, \mathrm{C}, \mathrm{G}$, and $\mathrm{T})$ junctions at the optimal configurations.

then $T_{\mathrm{pAp}}$ at $1.07 \mathrm{eV}$. In the region $[1.1,1.8] \mathrm{eV}$, the order is now $T_{\mathrm{pTp}}>T_{\mathrm{pCp}}>T_{\mathrm{pAp}}>T_{\mathrm{pGp}}$, which roughly reflects the energy order of the various LUMOs (see Table II). Such a rather sensitive energy dependence of the order of the transmission coefficients for the various molecules reflects the fact that the nucleotides are electronically rather similar. However, we will show that the $I-V$ curves offer a reasonable voltage range where the molecules can be distinguished.

The calculated $I-V$ curves in the moderate bias range $[-1,1]$ volt are presented in Fig. 11. In this particular bias window, the transport is well within the tunneling regime, since there are no peaks in the transmission attributable to any $\mathrm{p} X \mathrm{p}$ molecular orbitals (see Fig. 10). Importantly, in this moderate bias region, the order of the currents amplitude is the same as that of the transmission coefficients at the Fermi level, namely, $I_{\mathrm{pAp}}>I_{\mathrm{pGp}}>I_{\mathrm{pCp}}>I_{\mathrm{pTp}}$. This is persistent throughout the entire bias values investigated, suggesting that a current measurement in this range should be able to distinguish

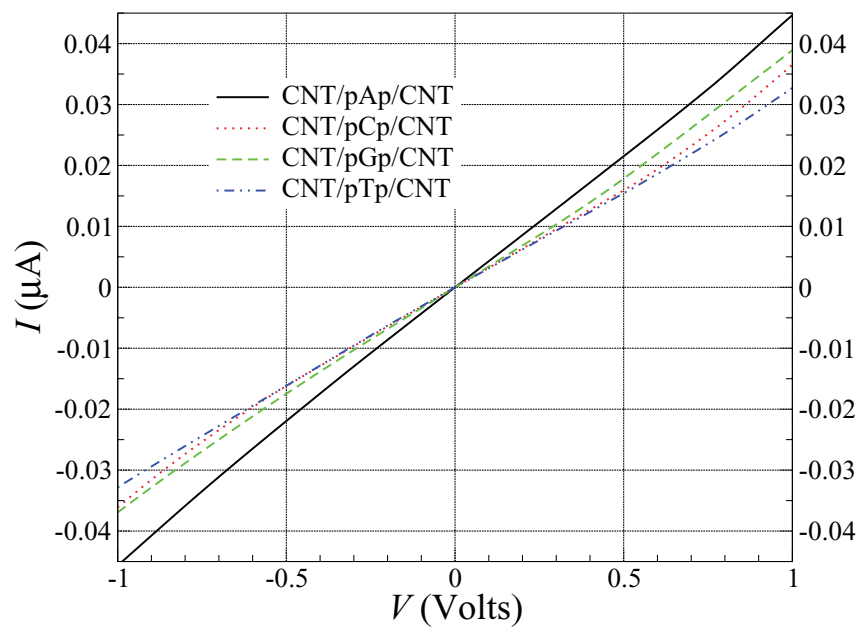

FIG. 11. (Color online) $I-V$ characteristics for the $\mathrm{CNT} / \mathrm{p} X \mathrm{p} / \mathrm{CNT}(X=\mathrm{A}, \mathrm{C}, \mathrm{G}$, and $\mathrm{T}$ ) junctions at the optimal configurations in the $[-1,1]$ volt bias range.

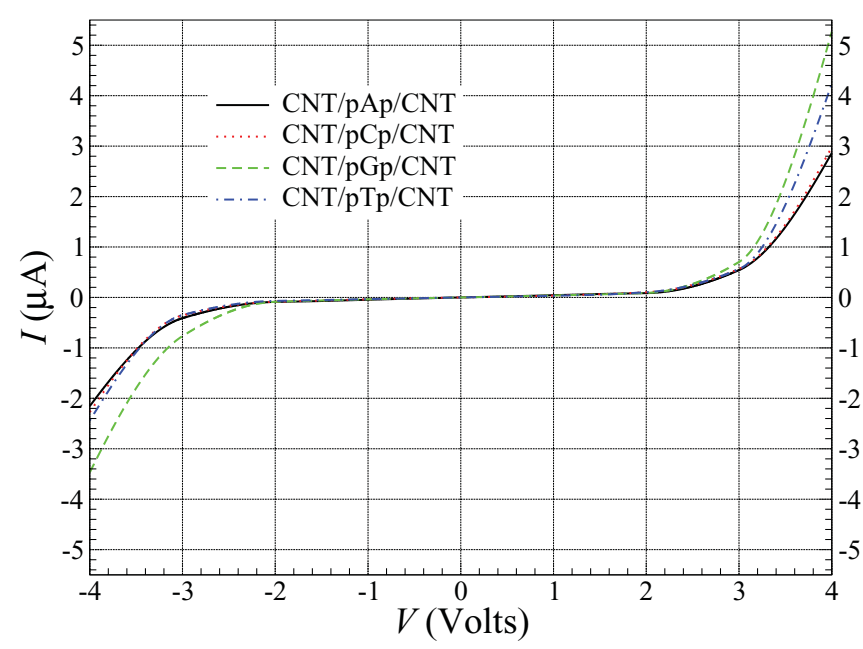

FIG. 12. (Color online) $I-V$ characteristics for the $\mathrm{CNT} / \mathrm{p} X \mathrm{p} / \mathrm{CNT}(X=\mathrm{A}, \mathrm{C}, \mathrm{G}$, and $\mathrm{T})$ junctions at the optimal configurations in the $[-4,4]$ volt bias range.

between the nucleotides. Furthermore, the current curves in Fig. 11 are well spaced when the bias is between 0.7 and $1 \mathrm{~V}$, a bias interval that therefore emerges as the ideal probing voltage range. We note that recently Tsutsui $e t$ al. reported a similar trend, namely, $I_{\mathrm{G}}>I_{\mathrm{C}}>I_{\mathrm{T}}$, for the peak currents of single nucleotides measured by two gold nanoelectrodes at a constant dc bias of $0.75 \mathrm{~V} .^{10}$

In Fig. 12, the $I-V$ curves for the same configurations are shown over an extended bias window ranging now from -4 to $4 \mathrm{~V}$. From the figure, we can clearly identify the transition from tunneling to a resonant transport regime at about $\pm 3 \mathrm{~V}$ for all nucleotides. This is the voltage that coincides with a drastic increase of the current. For voltages larger than 3.5 $\mathrm{V}$ and smaller than $-3.5 \mathrm{~V}$, the current signals associated to the different nucleotides have a steady order, namely, $I_{\mathrm{pGp}}>I_{\mathrm{pTp}}>I_{\mathrm{pCp}} \sim I_{\mathrm{pAp}}$ (see Fig. 12). Also notable is the fact that the $I-V$ characteristics are approximately symmetric with respect to the bias polarity, since the nucleotides are placed in the middle of the gap between the CNT electrodes.

In concluding, this section we should spend some words on discussing the potential effects of a high-bias measurement on the structural integrity of the nucleobases. In general, one may expect irreversible electrochemical changes of the DNA structure as the voltage is ramped up. Experimental data suggest that electrochemical voltages below $1 \mathrm{~V}$ are enough. ${ }^{35}$ This, however, requires electron charging of the DNA strand, a situation that is certainly encountered in longitudinal electron transfer but that in principle is not present in the tunneling transport investigated here. Furthermore, as the electrodeelectrode distance is rather narrow and it is just sufficient for the nucleobases to pass through, it will be extremely unlikely for any nucleobase to share the region of high electric field between the electrodes with either a counter-ion or a water molecule. This means that the efficiency of the electrochemical activity in our proposed device may be different from that of a conventional electrochemical experiments involving longitudinal transport. We note, however, that our results indicate that, in general, the nucleobases can be distinguished even at very small biases. 


\section{E. Transmission profile of nucleotides along the translocation path}

In the discussion presented in the previous section, each of the four nucleotides is placed at the optimal configuration in the corresponding $\mathrm{CNT} / \mathrm{p} X \mathrm{p} / \mathrm{CNT}(X=\mathrm{A}, \mathrm{C}, \mathrm{G}$, and $\mathrm{T})$ junction. We now examine the evolution of the transport properties of the nucleotides when they translocate through the gap between the CNT electrodes along the $x$ axis. This is precisely the motion that the nucleotides will undergo in a nanopore experiment (see Fig. 1). For simplicity, the $\mathrm{p} X \mathrm{p}$ molecules, which have limited conformational flexibility near the $6.4-\AA$-wide electrode gap, are assumed to be rigid. Their positions are measured as the distance between the nucleotide center of mass and the midpoint of CNT electrodes.

Figure 13 shows the profiles of the transmission coefficient at the Fermi level, $T\left(E_{\mathrm{F}}\right)=T_{0}$, along the nucleotides' translocation paths, with the peaks appearing at the optimal configurations [see Table I]. As already mentioned in Sec. III B, one can clearly see that the profiles of $\mathrm{pCp}$ and $\mathrm{pTp}$ are distinguishable from those of $\mathrm{pAp}$ and $\mathrm{pGp}$ by having one more pronounced transmission peak. The double-peak structure arises from the two nearly degenerate optimal configurations, that both pAp and pGp possess because of their different symmetry. Since both the cytosine and the thymine base can be flipped before entering the electrode gap, the order and the relative magnitude of these two peaks can be reversed. In addition, we also note that the profile of pGp appears to have a broad shoulder, indicative of a second high transmission configuration along the translocation path. From Fig. 13, we also observe that the transmission coefficient decays sharply once the nucleotides is moved outside the electrodes gap. Notably, the radius of the $(6,6)$ CNT is about $4.1 \AA$ while the distance between adjacent nucleotides in a stretched ssDNA is about $7 \AA$. As the tails of the transmission coefficient curves are quite small when $x<-3.5 \AA$ or $x>3.5 \AA$ (i.e., when the nucleobase is outside of electrodes gap), we conclude that having nucleotides in a sequence should not affect much the reading of the tunneling current of a single nucleotide.

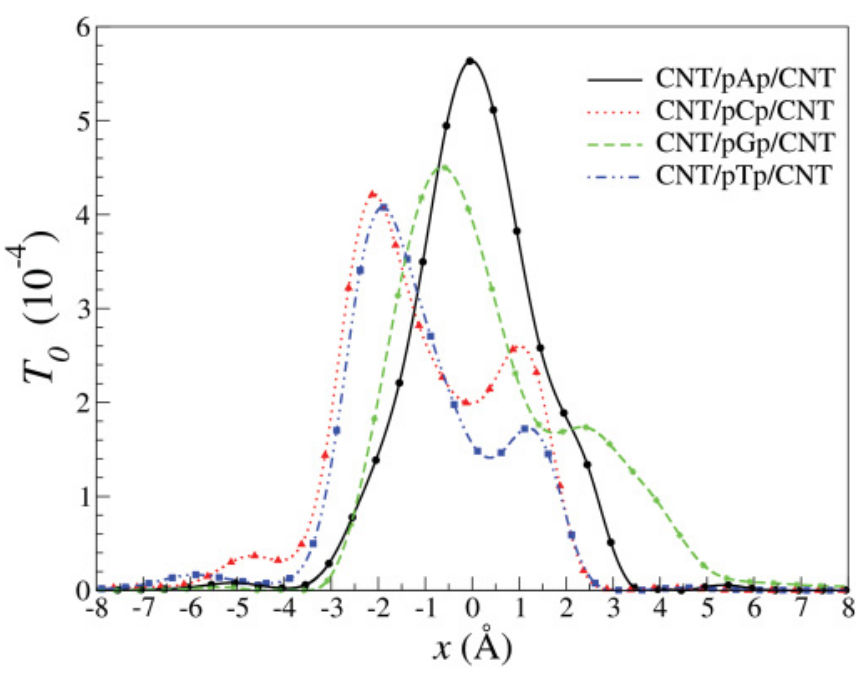

FIG. 13. (Color online) Transmission coefficient at the Fermi level along the DNA translocation path.
TABLE III. Aggregate transmission coefficient at the Fermi level along the DNA translocation path centered in the middle plane between the CNT electrodes (unit: $10^{-4} \AA$ ).

\begin{tabular}{lcccc}
\hline \hline & $\mathrm{pAp}$ & $\mathrm{pCp}$ & $\mathrm{pGp}$ & $\mathrm{pTp}$ \\
\hline $\int_{-3.5}^{3.5} T_{0} d x$ & 17.03 & 14.06 & 15.81 & 12.30 \\
$\int_{-8.0}^{8.0} T_{0} d x$ & 17.24 & 14.93 & 17.19 & 12.82 \\
\hline \hline
\end{tabular}

We next integrate $T_{0}$ along the translocation path to obtain the aggregate transmission coefficient (denoted as $A T$ ). Our results are summarized in Table III. $A T$ over the path $-3.5<x<3.5 \AA$ accounts for $99 \%, 94 \%, 92 \%$, and $96 \%$ of the $A T$ calculated over the more extended path $-8<x<$ $8 \AA$, respectively, for $\mathrm{pAp}, \mathrm{pCp}, \mathrm{pGp}$, and $\mathrm{pTp}$. This is a further indication that most of the current is collected when the nucleobase is within the CNT region and that adjacent nucleobases are far enough not to affect the measurement. Similar conclusions have been reached ${ }^{4}$ for Au electrodes at separations smaller than $1 \mathrm{~nm}$.

The aggregate transmission coefficient represents the timeaverage low-bias conductance over the translocation of each nucleotide, once we assume that the translocation occurs at a constant velocity. Interestingly, we find a well-spaced order in the $A T$, namely, $A T_{\mathrm{pAp}}>A T_{\mathrm{pGp}}>A T_{\mathrm{pCp}}>A T_{\mathrm{pTp}}$, both when the $A T$ is calculated within \pm 3.5 or $\pm 8 \AA$ from the electrode gap center. Notably, the order of the integrated transmission corresponds to the order found for the transmission at the optimal positions, which indeed dominate the transport during the translocation. More importantly, this fact indicates that the $\mathrm{pAp}>\mathrm{pGp}>\mathrm{pCp}>\mathrm{pTp}$ order is recurrent in most of the transport measurements we have described and therefore it is a rather robust result of the geometry investigated here.

\section{F. Effect of molecular configurations on the transport properties}

In the previous discussion about the transmission profiles, we have assumed that the nucleotides translocate ideally along the $x$ direction without any lateral displacement within the electrodes gap (along the $y$ axis). We now examine how $T_{0}$ varies when the nucleobases position fluctuates along the $y$ direction, by using the nucleobase center of mass of the relative optimal configuration as origin, $y=0$. As it can be seen in Fig. 14, $T_{0}$ decays quickly when the nucleobases move downward away from the electrodes (negative $y$ ). This is similar to what we have observed in Fig. 13 for a longitudinal displacement. Note that there is not much room for positive $y$ displacement now as this will need the nonplanar sugar ring and phosphate groups to enter the narrow electrodes gap. As such we stop the calculations at $y=1.5 \AA$, which corresponds to the largest displacement possible along that direction.

Next, we consider the effect of rotations of the $\mathrm{p} X \mathrm{p}$ molecules about the $z$ axis. As shown in Fig. 5, at the optimal configurations, the six-member ring of the end cap in each of the CNT electrodes is parallel to the plane of the nucleobases. Moreover, the cap ring tends to align with the pyrimidine rings of the purine bases (adenine and guanine) so that the corresponding hexagon edges are parallel to each other. In 


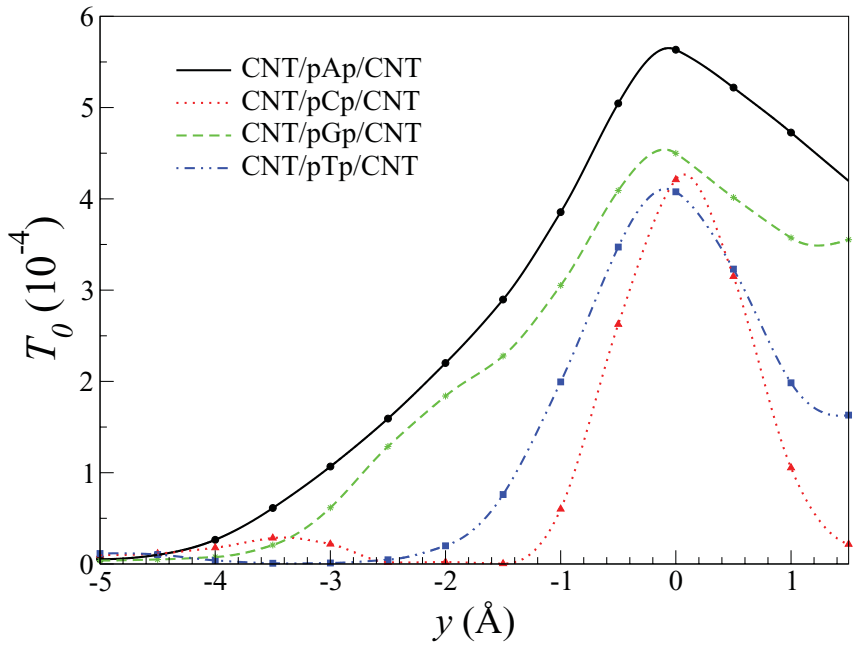

FIG. 14. (Color online) Transmission coefficient at Fermi level for the $\mathrm{CNT} / \mathrm{p} X \mathrm{p} / \mathrm{CNT}(X=\mathrm{A}, \mathrm{C}, \mathrm{G}$, and $\mathrm{T})$ junctions as the nucleotides move along the $y$ axis.

contrast, the edges are staggered with the pyrimidine rings of cytosine and thymine so that one of its vertex atoms faces the center of the pyrimidine. One may then expect that rotations may produce significant change in the low-bias conductance. This is however not the case. In fact, by plotting $T_{0}$ as a function of the rotation angle $\theta$ (see Fig. 15), we find only small changes, and most importantly also that the order of the amplitudes of the conductance of the various nucleobases does not change. This essentially means that rotations about the optimal configurations will not undermine our ability to identify the nucleobases.

Finally, we investigate the effects of possible fluctuations along the $z$ direction. First, we look at the transport as a function of the gap size between the CNT electrodes by plotting $T_{0}$ as function of the electrode distance, $d_{\mathrm{CNT}-\mathrm{CNT}}$ (see Fig. 16). In this case, we maintain the position of the plane containing the $\mathrm{p} X \mathrm{p}$ molecules fixed at the midpoint between

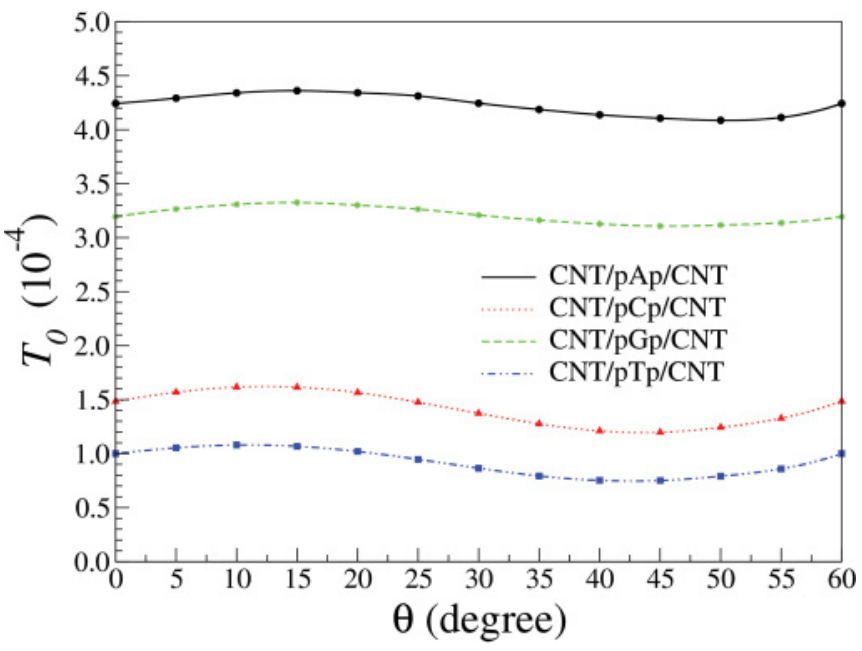

FIG. 15. (Color online) Transmission coefficient calculated at the Fermi level for the CNT/p $X \mathrm{p} / \mathrm{CNT}(X=\mathrm{A}, \mathrm{C}, \mathrm{G}$, and $\mathrm{T})$ junctions as a function of the rotation of angle of the $\mathrm{p} X \mathrm{p}$ molecules about the $z$ axis (vertical)

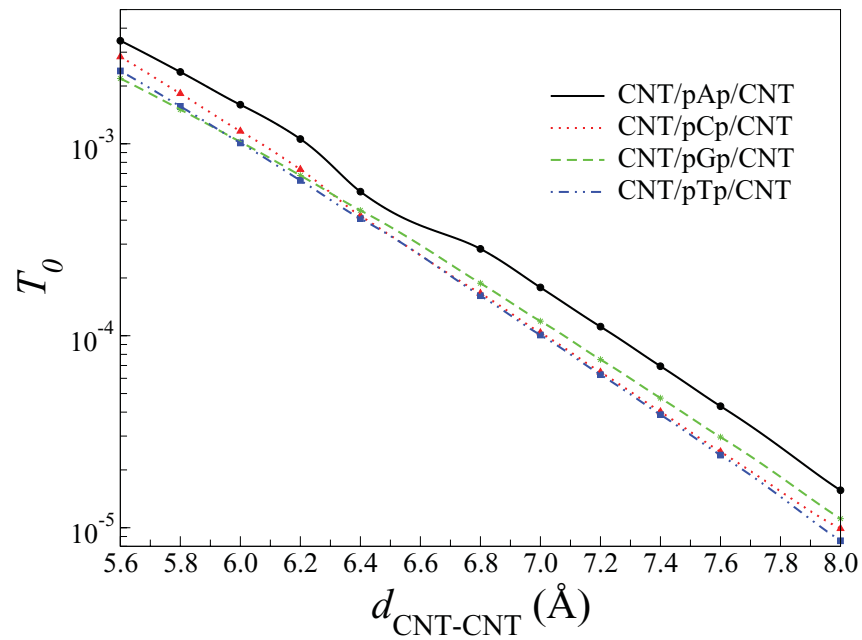

FIG. 16. (Color online) Transmission coefficient at Fermi level for the $\mathrm{CNT} / \mathrm{p} X \mathrm{p} / \mathrm{CNT}(X=\mathrm{A}, \mathrm{C}, \mathrm{G}$, and $\mathrm{T})$ junctions as a function of the electrode separation $d_{\mathrm{CNT}-\mathrm{CNT}}$. Note that $T_{0}$ decays exponentially when $d_{\mathrm{CNT}-\mathrm{CNT}}$ increases.

the electrodes. As expected from a tunneling process, we find that the transmission coefficient decreases exponentially with increasing $d_{\mathrm{CNT}-\mathrm{CNT}}$. Figure 16 also shows that the exponential decay rate $\alpha$, defined as the slope of the $\ln T(d)$ curves, is rather similar among the nucleotides. Interestingly, $T_{0}$ for $\mathrm{pAp}$ is always well separated from that of the other nucleotides for all the electrode gap distances investigated. In contrast, the relative order between $\mathrm{pCp}, \mathrm{pGp}$, and $\mathrm{pTp}$ depends on $d_{\text {CNT-CNT }}$. It then emerges that it is absolutely crucial to maintain the electrode separation as stable as possible during a measurement and also that this should be the smallest possible, so that configurational fluctuations will be highly suppressed.

Next and most importantly, we look at possible fluctuations of the position of the various $\mathrm{p} X \mathrm{p}$ molecules along the $z$ axis, i.e., we look at the possibility that a molecule moves closer to one of the two electrodes than to the other. In this case, we consider the electrode-electrode separation fixed at its optimal value and calculate both the total energy and $T_{0}$ as a function of the displacement of the $\mathrm{p} X \mathrm{p}$ molecule from the electrodes' gap mid position. Our results are reported in Fig. 17. Clearly, the midpoint between the electrodes is the position displaying the larger conductance. This decays as the molecule moves to the side. For an identical displacement (all the $\mathrm{p} X \mathrm{p}$ molecules are moved by the same amount), the order of the conductances remain unchanged. However, different bases at different vertical positions may show identical conductances. As such, if the vertical position during the translocation is not uniform, one may have difficulties in distinguishing a basis from another by simply measuring the zero-bias conductance. Importantly, our total energy calculations (bottom panel of Fig. 17) show that it is relatively energetically costly to have large displacement along the vertical axis ( $z$-axis), and that the midpoint corresponds to the energy minimum. This is a consequence of our narrow electrodes gap, and we expect that for larger electrode-electrode separation the midpoint will not be any longer the most favorable position. In this case, larger fluctuations are expected on an overall lower 


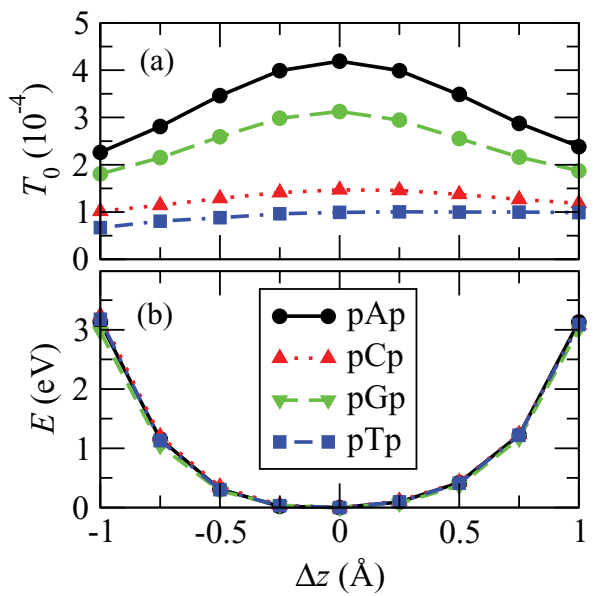

FIG. 17. (Color online) (a) Transmission coefficient at Fermi level for the $\mathrm{CNT} / \mathrm{p} X \mathrm{p} / \mathrm{CNT}(X=\mathrm{A}, \mathrm{C}, \mathrm{G}$, and $\mathrm{T}$ ) junctions as a function of the displacement along the $z$ axis, $\Delta z$. Here, $\Delta z=0$ corresponds to the electrodes' gap midpoint and the electrodes' separation is at its optimal value of $6.4 \AA$. In (b), we show the total energy as a function of $\Delta z$. Note that the energy minimum is at midpoint for all the four nucleotides.

conductance (see Fig. 16), and the detection will become not viable.

\section{G. DNA sequencing protocol}

With all the results of the previous sections in mind, we can now formulate a possible protocol for reading the nucleotide sequence. The proposed device functions by translocating ssDNA through a nanopore and simultaneously by measuring the transverse current between two closely spaced CNT nanotubes $[(6,6)$ close-ended CNTs at $6.4 \AA$ separation in our case]. The DNA strand can be pulled through the nanopore mechanically by an optical or magnetic tweezer ${ }^{36,37}$ with an approximately constant velocity. Typical velocities for optical tweezers have been reported ${ }^{36}$ at around $30 \mathrm{~nm} \mathrm{~s}^{-1}$. This means that one needs a time resolution in the $\mathrm{kHz}$ range in order to obtain a spatial resolution of $0.1 \AA$, which is certainly enough for detecting accurately the transmission profiles of the nucleotides shown in Fig. 13. Note that the currents involved are of the order of $10 \mathrm{nA}$ and we need an amplitude resolution of the order of $1 \mathrm{nA}$ or less (depending on the bias) for typical resistances of the order of $10 \mathrm{M} \Omega$. These are rather standard demands for conventional electronics. However, one has also to consider that fast fluctuations in the nucleobases positions are still possible ${ }^{38}$ and that these can be hardly controlled by optical tweezers. Whether or not the sequencing is possible will then depend on whether or not such fluctuations are small in amplitude at the time scale of the transport measurement.

In contrast, if the ssDNA translocation is electrophoretically driven, then the velocity may fluctuate considerably so that the recognition of the current profiles may become difficult. Furthermore, the typical translocation velocities are much larger than those achievable by mechanical manipulations. For instance, early studies ${ }^{39}$ reported speeds of the order of $10^{6} \mathrm{~nm} \mathrm{~s}^{-1}$, which require $\mathrm{GHz}$ time resolution for a subangstrom spatial resolution. More recently, a substantial slowing down has been achieved by gate modulation of the nanopore wall surface charges, with reported velocities of $55 \mu \mathrm{m} \mathrm{s}^{-1}$. This translates in a $5-\mathrm{MHz}$ time resolution for $0.1-\AA ̊$ spatial resolution. Clearly, $\mathrm{GHz}$ frequencies are out of the reach, unless the typical resistances are in the $\mathrm{k} \Omega$ range, while $\mathrm{MHz}$ resolution may be possible. In any case, we believe that for a usable device setup electrophoretically driven translocation is not suitable at present, at least if the signal is made of the current profiles during translocation.

Further information, however, can be extracted by simply taking the integral over time of each current peak and/or successive peaks. These are acquired during the translocation of an entire nucleobase across the gap between the electrodes. As such the time resolution required is now about two orders of magnitude larger than that needed for resolving the profiles. This means operating the device at $\sim 100 \mathrm{kHz}$ frequencies for slow electrophoretically driven translocations.

Finally, an alternative quantity to measure is, as proposed first by Zwolak et al., ${ }^{4}$ the statistical distribution of the current over time. This is a measure probably achievable only with the tweezers setup and essentially consists in holding the ssDNA at a fixed position and then, after the system has thermally equilibrated inside the nanopore, in measuring the current. We expect that the details of the current distributions may be device dependent, but the peak currents of the nucleobases should maintain the order $I_{\mathrm{pAp}}>I_{\mathrm{pGp}}>I_{\mathrm{pCp}}>I_{\mathrm{pTp}}$, at least in the suitable low-bias range around [0.7,1] V.

The statistical distributions of the transmission coefficient/current of the $\mathrm{CNT} / \mathrm{p} X \mathrm{p} / \mathrm{CNT}$ junctions are yet to be calculated for our device (see Fig. 1). However, some insights can be obtained by plotting the $T_{0}$ together with the interaction energy between the $\mathrm{p} X \mathrm{p}$ molecules and the CNT electrodes along the translocation path. The interaction

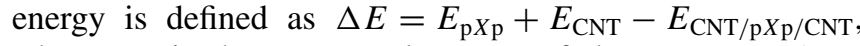
where $E_{\alpha}$ is the DFT total energy of the $\alpha$ system $(\alpha=$ $\mathrm{p} X \mathrm{p}, \mathrm{CNT}$ and $\mathrm{CNT} / \mathrm{p} X \mathrm{p} / \mathrm{CNT}$ ). Our results are shown in Fig. 18. The calculated interaction energies are one order of magnitude larger than that for typical hydrogen bonding $(0.1-0.4 \mathrm{eV})$. This is due to the strong $\pi-\pi$ interaction found in our device at the optimal configurations. Interestingly, we find
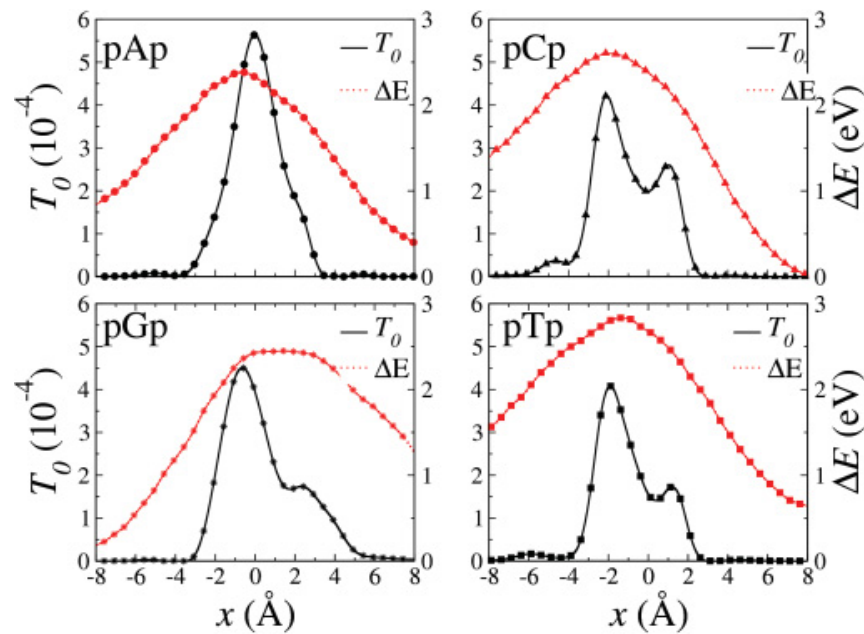

FIG. 18. (Color online) Transmission coefficient at the Fermi level against the interaction energy for the CNT/p $X \mathrm{p} / \mathrm{CNT}(X=\mathrm{A}$, $\mathrm{C}, \mathrm{G}$, and $\mathrm{T}$ ) junctions along the DNA translocation path. 
that the position of the maximum transmission corresponds approximately to the position displaying the largest interaction energy. As such, one may expect that when a nucleobase passes through the device, it will tend to remain between the electrodes and parallel to the end caps of CNTs. In other words, the same interaction responsible for the relatively large currents and for the suppression of the geometry fluctuations also acts to slow down the DNA translocation across the nanopore.

\section{CONCLUSIONS}

In summary, we have proposed a device for high throughput DNA nanopore sequencing, based on reading the transverse current across the nucleobases of ssDNA as it passes between two closely spaced single-walled close-ended $(6,6)$ CNTs. The device operation has been investigated theoretically with state of the art density functional theory combined with nonequilibrium quantum transport. In general, the electron transport through the various nucleobases can be roughly separated into a tunneling regime at low bias and a resonant transport one at higher bias. We have identified the optimal position of the various nucleotides yielding the highest zero-bias conductance and used them to analyze the various parameters affecting the device. In particular, we have focused our attention on the dependence of the electron transmission on the longitudinal position of the nucleotides with respect to the center of the electrodes, on possible lateral displacements and on the electrode separation.

The current/conductance profiles of the nucleotides along the DNA translocation path reveal that the pyrimidine bases (cytosine and thymine) have a two peaks structure, indicative of two high-transmission geometries. The same is not found for the other two bases. Importantly, we have found that the peak tunneling currents of the nucleobases have the following order $I_{\mathrm{pAp}}>I_{\mathrm{pGp}}>I_{\mathrm{pCp}}>I_{\mathrm{pTp}}$ at low bias and that the same order is maintained for the aggregate transmission coefficients. This order is the same reported for Au electrodes and a vertical arrangement of the nucleobases in the pore. The reason for such "universal" result is rooted in the position of the bases' HOMO with respect to the electrodes' Fermi level and of the decay of the nucleobases' wave functions into the vacuum. Both these features do not change when going from $\mathrm{Au}$ electrodes to CNTs. Furthermore, we have demonstrated that at this close electrode separation the current drops drastically as the nucleotides exit the electrodes region, so that well separated current signals should be detected during the translocation.

Finally, we have proposed a measurement protocol for rapid DNA sequencing. This is presently suitable for tweezer driven translocation and consists of three independent measurements. The first involves the pattern recognition of the current profile during the translocation of the nucleobases. The second relies on the fact that the aggregate transmission coefficient (and hence the integral of the current) along the translocation path has a well-defined order. Finally, the third concerns the time-distribution of the current for mechanically stabilized junctions, where the nucleotide to measure is held at a fixed position.

\section{ACKNOWLEDGMENTS}

X.C. gratefully acknowledges the European Community's Seventh Framework Programme for financial support (Project: nanoDNAsequencing: NanoTools for Ultra Fast DNA Sequencing; Grant agreement No. 214840). Additional financial support has been provided by (C.D.P. and S.S.) Science Foundation of Ireland (Grant No. 07/IN.1/I945) and (I.R., U.S., and S.S.) by the King Abdullah University of Science and Technology (ACRAB project). Calculations have been performed on the Shaheen supercomputer at the King Abdullah University of Science and Technology. Illuminating discussion with Nadjib Baadji are kindly acknowledged.
${ }^{1}$ J. J. Kasianowicz, E. Brandin, D. Branton, and D. W. Deamer, Proc. Natl. Acad. Sci. USA 93, 13770 (1996).

${ }^{2}$ D. Branton, D. W. Deamer, A. Marziali, H. Bayley, S. A. Benner, T. Butler, M. Di Ventra, S. Garaj, A. Hibbs, X. Huang, S. B. Jovanovich, P. S. Krstic, S. Lindsay, X. S. Ling, C. H. Mastrangelo, A. Meller, J. S. Oliver, Y. V. Pershin, J. M. Ramsey, R. Riehn, G. V. Soni, V. Tabard-Cossa, M. Wanunu, M. Wiggin, and J. A. Schloss, Nat. Biotechnology 26, 1146 (2008).

${ }^{3}$ M. Zwolak and M. Di Ventra, Rev. Mod. Phys. 80, 141 (2008).

${ }^{4}$ M. Zwolak and M. Di Ventra, Nano Lett. 5, 421 (2005).

${ }^{5}$ I. Rungger, X. Chen, U. Schwingenschlögl, and S. Sanvito, Phys. Rev. B 81, 235407 (2010).

${ }^{6}$ C. D. Pemmaraju, I. Rungger, X. Chen, A. R. Rocha, and S. Sanvito, Phys. Rev. B 82, 125426 (2010).

${ }^{7}$ J. Lagerqvist, M. Zwolak, and M. Di Ventra, Nano Lett. 6, 779 (2007).

${ }^{8}$ J. Lagerqvist, M. Zwolak, and M. Di Ventra, Biophys. J. 93, 2384 (2007).

${ }^{9}$ M. Krems, M. Zwolak, Y. V. Pershin, and M. Di Ventra, Biophys. J. 97, 1990 (2009).
${ }^{10}$ M. Tsutsui, M. Taniguchi, K. Yokota, and T. Kawai, Nat. Nanotechnology 5, 286 (2010).

${ }^{11}$ B. C. Gierhart, D. G. Howitt, S. J. Chen, Z. Zhu, D. E. Kotecki, R. L. Smith, and S. D. Collins, Sensors and Actuators B: Chemical 132, 593 (2008).

${ }^{12}$ X. Liang and S. Y. Chou, Nano Lett. 8, 1472 (2008).

${ }^{13}$ Z. Jiang, M. Mihovilovic, J. Chan, and D. Stein, J. Phys. Condens. Matter 22, 454114 (2010).

${ }^{14}$ P. Spinney, S. Collins, R. Smith, and D. Howitt, Sensors, 2009 IEEE, 37 (2009).

${ }^{15}$ A. P. Ivanov, E. Instuli, C. M. McGilvery, G. Baldwin, D. W. McComb, T. Albrecht, and J. B. Ede, Nano Lett. 11, 279 (2011).

${ }^{16}$ R. Zikic, P. S. Krstić, X.-G. Zhang, M. Fuentes-Cabrera, J. Wells, and X. Zhao, Phys. Rev. E 74, 011919 (2006).

${ }^{17}$ J. Lagerqvist, M. Zwolak, and M. Di Ventra, Phys. Rev. E 76, 013901 (2007).

${ }^{18}$ R. Zikic, P. S. Krstić, X.-G. Zhang, M. Fuentes-Cabrera, J. Wells, and X. Zhao, Phys. Rev. E 76, 013902 (2007).

${ }^{19}$ X.-G. Zhang, P. S. Krstić, R. Zikic, J. C. Wells, and M. FuentesCabrera, Biophys. J. 91, L04 (2006). 
${ }^{20}$ H. He, R. H. Scheicher, R. Pandey, A. R. Rocha, S. Sanvito, A. Grigoriev, R. Ahuja, and S. P. Karna, Phys. Chem. Lett. 112, 3456 (2008).

${ }^{21}$ Y. He, L. Shao, R. H. Scheicher, A. Grigoriev, R. Ahuja, S. Long, Z. Ji, Z. Yu, and M. Liu, Appl. Phys. Lett. 97, 43701 (2010).

${ }^{22}$ T. Nelson, B. Zhang, and O. V. Prezhdo, Nano Lett. 10, 3237 (2010).

${ }^{23}$ J. Prasongkit, A. Grigoriev, B. Pathak, R. Ahuja, and R. H. Scheicher, Nano Lett. 11, 1941 (2011).

${ }^{24}$ V. Meunier and P. S. Krstić, J. Comput. Phys. 128, 41103 (2008).

${ }^{25}$ T. Ohshiro and Y. Umezawa, Proc. Natl. Acad. Sci. USA 103, 10 (2006).

${ }^{26}$ J. He, L. Lin, P. Zhang, and S. Lindsay, Nano Lett. 7, 3854 (2007).

${ }^{27}$ A. R. Rocha, V. García-Suárez, S. W. Bailey, C. J. Lambert, J. Ferrer, and S. Sanvito, Nat. Mater. 4, 335 (2005).

${ }^{28}$ A. R. Rocha, V. M. García-Suárez, S. Bailey, C. Lambert, J. Ferrer, and S. Sanvito, Phys. Rev. B 73, 085414 (2006).

${ }^{29}$ I. Rungger and S. Sanvito, Phys. Rev. B 78, 035407 (2008).
${ }^{30}$ J. M. Soler, E. Artacho, J. D. Gale, A. García, J. Junquera, P. Ordejón, and D. Sánchez-Portal, J. Phys. Condens. Matter 14, 2745 (2002).

${ }^{31}$ C. Toher, A. Filippetti, S. Sanvito, and K. Burke, Phys. Rev. Lett. 95, 146402 (2005).

${ }^{32}$ C. Toher and S. Sanvito, Phys. Rev. Lett. 99, 056801 (2007).

${ }^{33}$ C. Toher and S. Sanvito, Phys. Rev. B 77, 155402 (2008).

${ }^{34}$ I. Rungger, O. Mryasov, and S. Sanvito, Phys. Rev. B 79, 094414 (2009).

${ }^{35}$ T. Liu and J. K. Barton, J. Am. Chem. Soc. 127, 10160 (2005).

${ }^{36}$ U. F. Keyser, B. N. Koeleman, S. van Dorp, D. Krapf, R. M. M. Smeets, S. G. Lemay, N. H. Dekker, and C. Dekker, Nat. Phys. 2, 473 (2006).

${ }^{37}$ H. Peng and X. S. Ling, Nanotechnology 20 (2009).

${ }^{38}$ R. R. Johnson, A. Kohlmeyer, A. T. C. Johnson, and M. L. Klein, Nano Lett. 9, 537 (2009).

${ }^{39}$ D. Fologea, M. Gershow, B. Ledden, D. S. McNabb, J. A. Golovchenko, and J. Li, Nano Lett. 5, 1905 (2005). 